\title{
Diabetische Nierenerkrankung (Update 2019)
}

\section{Positionspapier der Österreichischen Diabetes Gesellschaft und der Österreichischen Gesellschaft für Nephrologie}

\author{
Harald Sourij · Roland Edlinger · Friedrich C. Prischl · Martin Auinger · Susanne Kaser · Sabine Horn · \\ Bernhard Paulweber - Alexandra Kautzky-Willer - Marcus Säemann · Rudolf Prager - Martin Clodi · \\ Guntram Schernthaner - Gert Mayer · Rainer Oberbauer · Alexander R. Rosenkranz
}

Online publiziert: 12. April 2019

(c) Der/die Autor(en) 2018

Zusammenfassung Rezente epidemiologische Untersuchungen zeigen, dass etwa $2-3 \%$ aller Österreicher Diabetes mit Nierenbeteiligung aufweisen. Diese betrifft somit in Österreich etwa 250.000 Menschen. Das Risiko des Auftretens und Fortschreitens der Erkrankung kann durch Lebensstilinterventionen und Optimierung der Einstellung des Blutdrucks, Blutzuckers und spezielle Medikamentenklassen vermindert werden. In diesem gemeinsamen Artikel der

\footnotetext{
H. Sourij $(\bowtie)$

Klinische Abteilung für Endokrinologie und Diabetologie, Universitätsklinik für Innere Medizin, Medizinische Universität Graz, Auenbruggerplatz 15, 8010 Graz, Österreich ha.sourij@medunigraz.at

R. Edlinger $\cdot$ M. Auinger $\cdot$ R. Prager

3. Medizinische Abteilung, Karl Landsteiner Institut für Stoffwechselerkrankungen und Nephrologie, Krankenhaus Hietzing der Stadt Wien, Wien, Österreich

F. C. Prischl

IV. Interne Abteilung/Bereich Nephrologie, Klinikum Wels-Grieskirchen, Wels, Österreich

S. Kaser

Universitätsklinik für Innere Medizin I, Medizinische

Universität Innsbruck, Innsbruck, Österreich

S. Horn

Abteilung für Innere Medizin, LKH Villach, Villach,

Österreich

B. Paulweber

Universitätsklinik für Innere Medizin I, Landeskrankenhaus

Salzburg, Uniklinikum der PMU, Salzburg, Österreich
}

Österreichischen Gesellschaften für Nephrologie und Diabetologie werden entsprechende Diagnostik und therapeutische Strategien bei diabetischer Nierenerkrankung vorgeschlagen.

Schlüsselwörter Diabetische Nierenerkrankung · Chronische Niereninsuffizienz • Diabetische Nephropathie - Albuminurie - Mikrovaskuläre Komplikationen
A. Kautzky-Willer

Klinische Abteilung für Endokrinologie und Stoffwechsel, Universitätsklinik für Innere Medizin III, Medizinische Universität Wien, Wien, Österreich

\section{Säemann}

6. Medizinische Abteilung mit Nephrologie \& Dialyse, Wilhelminenspital, Wien, Österreich

\section{Clodi}

Abteilung für Innere Medizin, Krankenhaus Barmherzige Brüder Linz, Linz, Österreich

\section{G. Schernthaner}

1. Medizinische Abteilung, Krankenhaus Rudolfstiftung, Wien, Österreich

\section{G. Mayer}

Nephrologie und Hypertensiologie, Universitätsklinik für Innere Medizin IV, Medizinische Universität Innsbruck, Innsbruck, Österreich

\section{R. Oberbauer}

Klinische Abteilung für Nephrologie und Dialyse, Universitätsklinik für Innere Medizin III, Medizinische Universität Wien, Wien, Österreich

\section{A. R. Rosenkranz}

Klinische Abteilung für Nephrologie, Universitätsklinik für Innere Medizin, Medizinische Universität Graz, Graz, Österreich 
Diabetic kidney disease (Update 2019) Position paper of the Austrian Diabetes Association and the Austrian Society for Nephrology

Summary Recent epidemiological investigations have shown that approximately $2-3 \%$ of all Austrians suffer from diabetes with renal involvement, i.e. 250,000 people in Austria are affected. The risk of occurrence and progression of this disease can be ameliorated by life style interventions as well as optimization of blood pressure, blood glucose levels and special drug classes. The present article represents the joint recommendations of the Austrian Diabetes Association and the Austrian Society for Nephrology for the diagnostics and treatment strategies of diabetic kidney disease.

Keywords Diabetic kidney disease - Chronic kidney disease · Diabetic nephropathy $\cdot$ Albuminuria $\cdot$ Microvascular complications

Diabetes mellitus und vaskuläre Erkrankungen stellen die häufigsten Ursachen terminalen Nierenversagens in Österreich dar [1]. Strategien zur Verhinderung des Auftretens bzw. der Progression sind daher von größter Bedeutung. Im Jahr 2017 waren laut österreichischem Dialyse- und Transplantationsregister (OEDTR) 25\% der Neuzugänge zur Dialyse Menschen mit Diabetes mellitus (23\% Typ-2-Diabetes [DM2], $2 \%$ Typ-1-Diabetes [DM1]). Es muss darauf hingewiesen werden, dass seit 2007 die inzidenten Zahlen der Dialysepatienten mit DM2 kontinuierlich rückläufig sind, die Prävalenz jedoch weiterhin steigt oder stabil bleibt [1]. Das Letztere wird durch rezente Daten aus dem OEDTR unterstrichen, die eine Steigerung des Überlebens von DM2-Patienten im Zeitraum von 1998 auf 2007 um insgesamt 1 Jahr zeigen konnten [2].

\section{Die Nierenerkrankung bei Patienten mit Diabetes mellitus 1 (DM1)}

Der Verlauf der Nierenerkrankung beim Patienten mit DM1 ist weniger variabel als bei Patienten mit DM2 und die optimale/intensivierte Blutzuckereinstellung ist hier die wichtigste Intervention. Bei optimaler Einstellung $\left(\mathrm{HbA}_{\mathrm{lc}}<7 \%(53 \mathrm{mmol} / \mathrm{mol})\right) \mathrm{kam}$ es nach 30 Jahren $\mathrm{zu}$ einer 36 - bis $76 \%$ igen Reduktion der mikrovaskulären Komplikationen im Vergleich zur Gruppe mit einem $\mathrm{HbA}_{\mathrm{lc}} \sim 9 \%$ [3]. Die Inzidenz der terminalen Niereninsuffizienz in der intensiv behandelten Gruppe lag bei 11/1000 Patienten [4]. Sobald entweder eine Hypertonie oder eine Albuminurie (ab Stadium A2) vorliegt, gilt die medikamentöse RAASBlockade als gesicherte Therapie [5, 6].

\section{Die Nierenerkrankung bei Patienten mit Diabetes mellitus 2 (DM2)}

Die Prävalenz des DM2 in Österreich schwankt regional zwischen 7 und 9\%, etwa $25 \%$ dieser Patienten haben auch eine Niereninsuffizienz $(\mathrm{CKD}=$ „chronic kidney disease“) Stadium G3 oder höher (eGFR $<60 \mathrm{ml} / \mathrm{min} / 1,73 \mathrm{~m}^{2}$ ) [7]. Rezente amerikanische Daten gehen ebenso davon aus, dass ca. $24 \%$ aller Fälle von chronischer Niereninsuffizienz (i.e. eGFR $<60 \mathrm{ml} / \mathrm{min} / 1,73 \mathrm{~m}^{2}$ oder Albumin/Kreatinin-Ratio $\geq 30 \mathrm{mg} / \mathrm{g}$ oder beides) nach Korrektur für demografische Faktoren auf das Konto des Diabetes mellitus gehen [8]. Durch das erhöhte Mortalitätsrisiko von DM2-Patienten („competing risk of death“) versterben viele Patienten, bevor sie das Stadium der terminalen Niereninsuffizienz erreichen, und somit sind nur ein Viertel aller Patienten mit terminaler Nierenerkrankung DM2-Patienten [1].

Die Nierenerkrankung bei DM2- ist heterogener als bei DM1-Patienten, und somit sind der Verlauf und die Prognose nur ungenau abzuschätzen. Gesichert ist bei DM2 die Wirksamkeit einer RAAS-Blockade sowohl hinsichtlich der Entstehung einer Albuminurie [9, 10] als auch hinsichtlich Progressionsverzögerung der diabetischen Nierenerkrankung, unabhängig vom Blutdruck (gemessen anhand der Albuminurie) [11, 12]. Neben der Reduktion einer manifester Nierenerkrankung konnte in der HOPE-Studie auch eine deutliche Mortalitätsreduktion (24\%) gezeigt werden [6].

Aufgrund der meist schon längeren Zeitspanne zwischen Beginn der gestörten Stoffwechsellage und Diagnose des Typ-2-Diabetes findet sich häufig zum Zeitpunkt der Diagnosestellung eine Albuminurie. Ohne spezielle Intervention entwickeln ca. 20-40\% der Patienten mit einer Albuminurie Stadium A2 (s. unten) eine größere Albuminurie bzw. Proteinurie (Stadium A3), die jedoch insgesamt nur bei etwa $20 \%$ der Patienten innerhalb von 20 Jahren zu einer terminalen Niereninsuffizienz fortschreitet [13]. Das Auftreten einer Albuminurie per se sowie das Vorliegen einer chronischen Niereninsuffizienz gehen mit einer erhöhten Inzidenz makrovaskulärer Komplikationen und Mortalität einher [14]. Früher ging man von einem „klassischen Durchlaufen“ aller Stadien bis zur Entwicklung der terminalen Niereninsuffizienz aus und betonte die Wertigkeit der Albuminurie im Stadium A2 als Parameter der Frühdiagnostik. Bei vielen diabetischen Patienten mit erhöhten Nierenfunktionsparametern findet sich jedoch keine Albuminurie [13], sodass hier primär eine mikro-/makrovaskuläre Komponente in der Niere anzunehmen ist, auch eine Regression der Albuminurie mit und ohne Therapie ist möglich bzw. ein unterschiedlicher pathogenetischer Mechanismus, der auch in Zukunft andere therapeutische Schritte als bisher erfordern könnte. 
Abb. 1 Stadieneinteilung der chronischen Niereninsuffizienz (a) und deren Risikokonstellation abhängig von der Stadieneinteilung unter Einbeziehung der Albuminurie (b). (Nach [33])

\begin{tabular}{|l|l|l|}
\hline eGFR $\left(\mathrm{ml} / \mathrm{min} / 1,73 \mathrm{~m}^{2}\right)$ & Stadium & Funktionseinschränkung \\
\hline$>90$ & G1 & keine \\
\hline $60-89$ & G2 & geringgradig \\
\hline $45-59$ & G3a & gering- bis mittelgradig \\
\hline $30-44$ & G3b & mittel- bis hochgradig \\
\hline $15-29$ & G4 & hochgradig \\
\hline$<15$ & G5 & Nierenversagen \\
\hline a & & \\
\hline
\end{tabular}

\begin{tabular}{|l|l|l|l|}
\hline & A1 & A2 & A3 \\
\hline G1 & & & \\
\hline G2 & & & \\
\hline G3a & & & \\
\hline G3b & & & \\
\hline G4 & & & \\
\hline G5 & & & \\
\hline
\end{tabular}

laut KDIGO Guidelines 2012

Grün: geringes Risiko, Mortalität, kardiovaskulär, Progression der CNE etc. Gelb: mäßiges Risiko, Orange: hohes Risiko, Rot: sehr hohes Risiko

b Albuminausscheidung in A1 ( $<30 \mathrm{mg} / \mathrm{g}$ Kreatinin), sowie A2 $(30-300 \mathrm{mg} / \mathrm{g})$ und A3 $(>300 \mathrm{mg} / \mathrm{g})$

\section{Spektrum der diabetischen Nierenerkrankung}

Mitte des 20. Jahrhunderts wurde der Begriff der diabetischen Nephropathie als klinisches Syndrom, basierend auf interkapillärer oder nodulärer Glomerulosklerose (Kimmelstiel-Wilson) bei Patienten mit Diabetesdauer länger als 20 Jahre, persistierender Albuminurie, Hypertonie, Retinopathie und progressivem Nierenfunktionsverlust geprägt [15, 16]. Diese Definition wurde später durch die klassischen 5 Stadien des natürlichen Krankheitsverlaufes ergänzt: Stadium 1, Hyperfiltration mit glomerulärer Hypertrophie und transienter Albuminurie; Stadium 2, die ruhige Phase mit Normalisierung der GFR und der Albuminexkretion, begleitet von Verdickung der glomerulären Basalmembran und einer Mesangialexpansion; Stadium 3, inzipiente Nephropathie mit persistierender Mikroalbuminurie; Stadium 4, offensichtliche Nephropathie mit Verschlechterung der Albuminurie (von Mikro- zur Makroalbuminurie) und Retinopathie, Blutdruckverschlechterung und Abnahme der Nierenfunktion; und letztlich Stadium 5 mit variablem GFR-Verlust bis zur terminalen Niereninsuffizienz, charakteristischerweise 25 bis 30 Jahre nach Krankheitsbeginn [17]. Obwohl dieses beschriebene Modell und der Ablauf primär auf Daten von Patienten mit DM1 basierte [18, 19], wurde es auch auf
Patienten mit DM2 angewandt und war auch noch zuletzt in den Richtlinien der beiden Gesellschaften so verankert [20]. Mittlerweile geht man davon aus, dass mehr als $50 \%$ der Patienten mit DM2 in Langzeitbeobachtungen eine GFR $<60 \mathrm{ml} / \mathrm{min}$ ohne vorangehende Albuminurie entwickeln [21-24] bzw. der Verlauf der Albuminurie nicht mit dem Nierenfunktionsverlust korreliert [25]. Ähnliche Beobachtungen gibt es auch für DM1 [26].

Der neue Begriff der „diabetischen Nierenerkrankung" reflektiert die beobachtete strukturelle und klinische Heterogenität sowohl bei DM1 als auch v. a. bei DM2. Daher erscheint der Begriff der „diabetischen Nierenerkrankung" wesentlich besser die unterschiedlichen Verläufe, speziell die vorhergehend beschriebene Diskordanz zwischen Abnahme der Nierenfunktion und Albuminurie, zu reflektieren [27], was bisher nicht entsprechend beachtet wurde. Dieser Begriff wurde daher auch im angloamerikanischen Bereich bereits eingeführt und von den entsprechenden Expertengruppen übernommen [28, 29].

Histologische Untersuchungen zeigten bei Nierenbiopsien von Patienten mit Albuminurie und DM2 3 pathologische Kategorien [30]: Kategorie I mit fast normaler Nierenstruktur, die bei $35 \%$ der Personen mit moderater Albuminurie (vormals Mikroalbuminurie) sowie $10 \%$ der Personen mit Proteinurie gese- 
hen wurden; Kategorie II hatte eine typische diabetische Nephropathologie (wie bei DM1) bei 30\% der Patienten mit moderater Albuminurie und 55\% mit Proteinurie. Zusätzlich fanden sich klinisch bei dieser Population auch eine signifikante proliferative Retinopathie, längeres Bestehen des DM2, schlechte metabolische Kontrolle und progredienter Nierenfunktionsverlust; bei Patienten in Kategorie III zeigten sich atypische Veränderungen des Nierengewebes und nur milde glomeruläre diabetische Läsionen in 35\% der Fälle sowohl bei moderater Albuminurie als auch Proteinurie [30]. In der Folge wurde von der Renal Pathology Society eine Klassifikation auf Basis von Biopsien von Patienten mit DM1 und 2 erstellt, die 4 Kategorien (Klasse I-IV) umfasst [31]. Diese neue Klassifikation, welche auch den Grad der interstitiellen Fibrose, interstitiellen Inflammation und vaskuläre Läsionen berücksichtigt, hat zum Ziel, Kriterien zu erstellen, welche sowohl über die Schwere der Läsionen wie über den prognostischen Wert informieren. Tatsächlich könnte dies in Zukunft zu einem wichtigen Tool hinsichtlich Zeitpunkt und Art von Therapien werden und somit das Outcome bei diabetischer Nierenerkrankung positiv beeinflussen [32].

Eine Stadieneinteilung, wie sie früher für die Definition diabetische Nephropathie auch in internationalen Leitlinien durchgeführt wurde, ist aus Sicht der Gesellschaften aufgrund der variablen Verlaufsform $\mathrm{zu}$ hinterfragen, weswegen sich die Gesellschaften entschlossen haben, auf die klassische CKD-Klassifikation von KDIGO (Kidney Disease - Improving Global Outcome) zurückzugreifen [33]. Diese unterteilt nach der geschätzten glomerulären Filtrationsrate (eGFR) in Stadien G1 bis G5 (Abb. 1a), unterteilt Stadium G3 in G3a (eGFR 45-59) und G3b (eGFR 30-44) und zusätzlich nach der Albuminausscheidung in A1 (<30 mg/g Kreatinin), A2 (30-300 mg/g) und A3 (>300 mg/g). Zusätzlich wird in der neuen Klassifizierung auch farblich das Mortalitätsrisiko dargestellt (Abb. 1b).

\section{Bestimmung der Nierenfunktion}

Zur Beurteilung des Ausmaßes der Nierenfunktionseinschränkung sollte eine der derzeit gängigen Schätzformeln verwendet werden, welche bereits in den meisten Labors implementiert sind. Eine ausschließliche Serumkreatininbestimmung ist v. a. bei älteren Menschen oft irreführend, da keine lineare Korrelation zur tatsächlichen Nierenfunktion besteht. Die mittels MDRD (Modification of Diet in Renal Disease)-Formel geschätzte glomeruläre Filtrationsrate (eGFR) ist für den Bereich zwischen 20 und $60 \mathrm{ml} / \mathrm{min} / 1,73 \mathrm{~m}^{2}$ für Personen über 18 Jahre validiert [34]. Die Basis der Berechnung soll eine nach IDMS („isotope dilution mass spectrometry“)-Goldstandard kalibrierte Serumkreatininbestimmung sein ([35]; Tab. 1). Rezent empfehlen die meisten Gesellschaften die CKD-EPI (Chronic Kidney Disease Epide-
Tab. 1 Darstellung der beiden am häufigsten verwendeten Schätzformeln zur Bestimmung der Nierenfunktion. (Nach [34, 36])

Glomeruläre Filtrationsrate berechnet (eGFR) - MDRD4-Formel
GFR $(\mathrm{ml} / \mathrm{min}) / 1,73 \mathrm{~m}^{2} \mathrm{KÖF}=186 \times\left(\mathrm{Scr}^{-1,154} \times\right.$ Alter- $0.203 \times(0,724$ bei
Frauen)
Glomeruläre Filtrationsrate berechnet $(\mathrm{eGFR})-\mathrm{CKD}$-EPI-Formel
$\mathrm{GFR}=141 \times \min \left(\mathrm{S}_{\mathrm{c} r} / \kappa, 1\right) \alpha \times \max \left(\mathrm{s}_{\mathrm{cr}} / \kappa, 1\right)-1,209 \times 0,993$ Alter $\times$
$1,018($ Frauen $) \times 1,159(\mathrm{Sch} w a r z e)$
KÖF Körperoberfläche, $s_{\mathrm{cr}}$ Serumkreatinin, Frauen: $\kappa=0,7 ; \alpha=-0,329$,
Männer: $\kappa=0,9 ; \alpha=-0,411$

Tab. 2 Stadien der Albuminurie. (Nach [33])

\begin{tabular}{|l|l|l|}
\hline $\begin{array}{l}\text { Albuminurie } \\
\begin{array}{l}\text { Normal }(<10) \text { bzw. minimal er- } \\
\text { höht }\end{array}\end{array}$ & $\begin{array}{l}\text { Stadium } \\
\text { A1 }\end{array}$ & $<30$ \\
\hline Mäßig erhöht & A2 & $30-300$ \\
\hline Stark erhöht & A3 & $>300$ \\
\hline
\end{tabular}

miology Collaboration)-Formel als Alternative ([36]; Tab. 1). Für diese Formel wurde mehrfach gezeigt, dass sie v. a. im Stadium 2-3 genauer als die MDRDFormel und somit besser zur Risikostratifizierung geeignet ist $[37,38]$.

Aufgrund der Ressourcen und Praktikabilität sind in der täglichen Praxis andere Schätzformeln z.B. unter Einbeziehung von Cystatin C derzeit nicht von Bedeutung. Zur besseren allgemeinen Verständlichkeit schlagen die Gesellschaften vor, die Nierenfunktion als \% Nierenfunktion $\mathrm{zu}$ interpretieren, was bei einem annähernden Normalwert von etwa $100 \mathrm{ml} / \mathrm{min} / 1,73 \mathrm{~m}^{2}\left(90-120 \mathrm{ml} / \mathrm{min} / 1,73 \mathrm{~m}^{2}\right)$ durchaus gerechtfertigt erscheint.

\section{Diagnostik der diabetischen Nierenerkrankung}

\section{Screening auf Albuminurie}

Bei DM1 sollte der Beginn des jährlichen Screenings auf Albuminurie erstmals 5 Jahre nach Diagnosestellung, bei DM2 mit der Diagnosestellung erfolgen. Generell wird empfohlen als Screening nur die Messung der Albumin/Kreatinin-Ratio aus dem Spontanharn durchzuführen [39]. Es soll an dieser Stelle nochmals darauf hingewiesen werden, dass unter anderem von der American Diabetes Association empfohlen wurde, unabhängig von der Bestimmung der Albuminurie auch eine regelmäßige eGFR-Bestimmung insbesondere bei DM1 mit zumindest 5 Jahren Laufdauer und bei allen DM2-Patienten zu veranlassen [40]. Des Weiteren wäre zu erwähnen, dass die bereits oben genannten KDIGO-Leitlinien empfehlen, den Begriff Mikroalbuminurie (entsprechend dem Stadium A2; moderate Albuminurie) nicht mehr länger zu verwenden, da diese Beschreibung irreführend ist (es handelt sich weder um ein kleines noch ein verändertes Albumin) [33]. Die vorgeschlagenen Kategorien A1-A3 drücken 


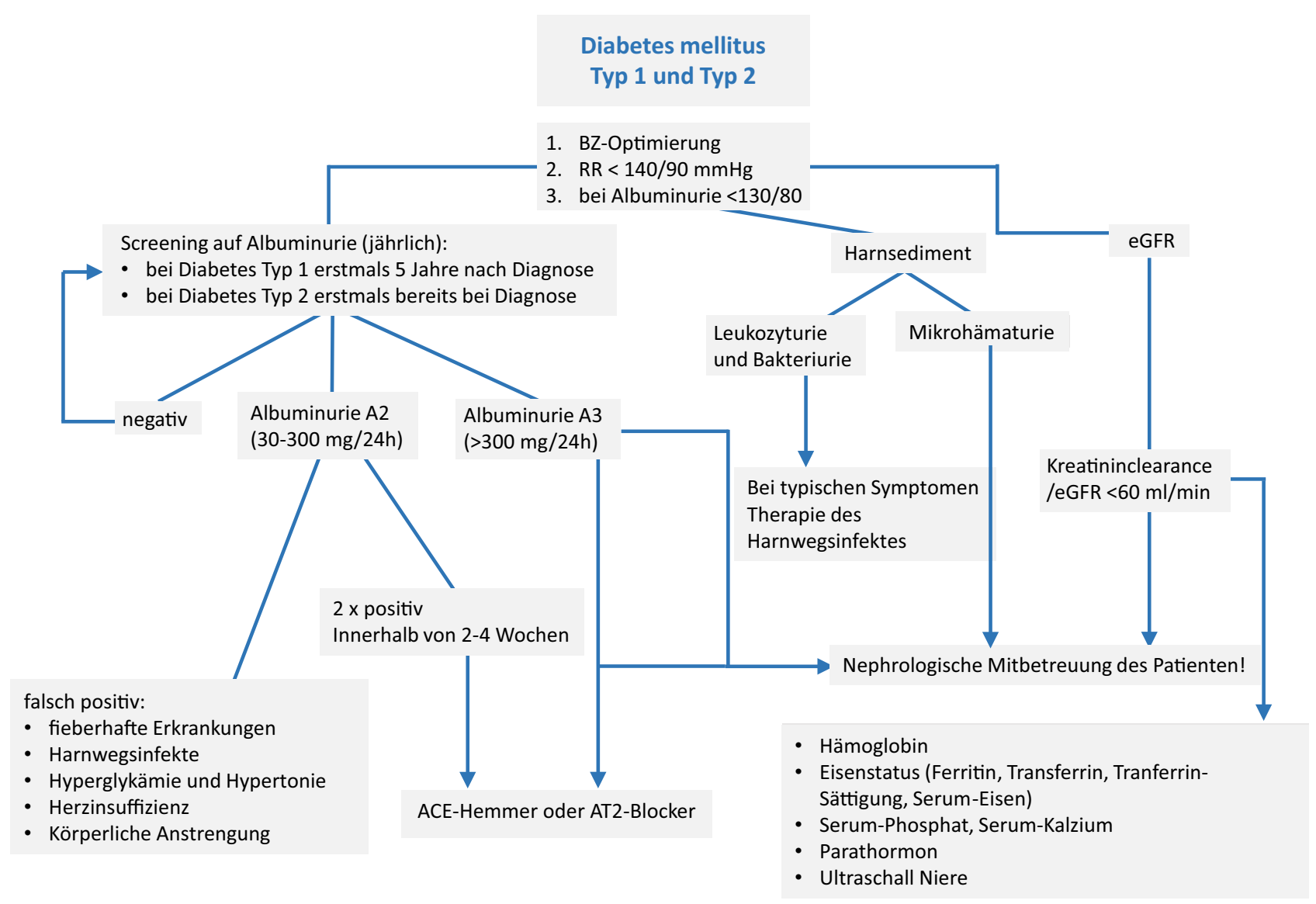

Abb. 2 Flowchart der initialen Abklärung einer möglichen diabetischen Nierenerkrankung

das Ausmaß der Albuminexkretion quantitativ besser aus (Tab. 2).

Aufgrund der Variabilität der Albuminurie wird zur Diagnostik der Albuminurie folgendes Vorgehen empfohlen: Es gilt die 2 aus 3 Regel: Wenn 2 hintereinander $\mathrm{zu}$ analysierende Urinproben übereinstimmend positiv oder negativ sind, ist eine Albuminurie bewiesen bzw. ausgeschlossen. Wenn eine Urinprobe negativ und die andere positiv ist, sollte eine dritte Urinprobe auf Albuminurie getestet werden. $\mathrm{Zu}$ beachten ist, dass positive Befunde z.B. auch bei akut fieberhaften Erkrankungen, Harnwegsinfekten und arterieller Hypertonie, bei Herzinsuffizienz und nach körperlicher Anstrengung aufgrund nichtdiabetischer Pathologien möglich sind. Aufgrund der besten Praktikabilität hat sich in den letzten Jahren die Bestimmung der Albumin/Kreatinin-Ratio bzw. bei Zunahme der Proteinurie die Protein/Kreatinin-Ratio im Harn zunehmend durchgesetzt. Ein konklusives Flussdiagramm zum Screening zeigt die Abb. 2.

\section{Differenzialdiagnosen bei Patienten mit diabeti- scher Nierenerkrankung}

Auch bei Diabetikern sollte immer an eine mögliche andere, nichtdiabetische Ursache der Proteinurie und/oder Nierenfunktionseinschränkung gedacht werden, insbesondere wenn mindestens eines der folgenden Kriterien erfüllt ist:

- Diabetesdauer unter 5 Jahren bei Typ-1-Diabetes,

- fehlende (insbesondere proliferative) diabetische Retinopathie als Ausdruck einer generalisierten diabetischen Mikroangiopathie,

- pathologisches Harnsediment mit Mikrohämaturie (insbesondere Akanthozytennachweis und Erythrozytenzylinder),

- sehr rasche Zunahme der Albuminurie, definiert als Klassenwechsel der Albuminurie (A1 auf A2 oder A2 auf A3 innerhalb eines Jahres),

- rascher Kreatininanstieg,

- Auffälligkeiten in der Nierensonographie (z.B. Seitendifferenz der Organgröße), welche an eine andere Nierenpathologie denken lassen.

Differenzialdiagnostisch häufig zu erwägende Nierenerkrankungen, die auch zusätzlich zu einer „diabetischen Nierenerkrankung " bestehen können, sind eine hypertensive oder eine ischämische Nephropathie als Folge einer Atherosklerose der größeren Nierengefäße. Bei ausgeprägter Albuminurie sind insbesondere andere renale Erkrankungen (u.a. Vaskulitiden, Glomerulonephritiden) anzudenken. Bei unklaren Befunden sollte großzügig im Rahmen der nephrologischen $\mathrm{Ab}$ - 
klärung die Indikation zur Nierenbiopsie gestellt werden.

\section{Therapeutische Gesichtspunkte bei Patienten mit diabetischer Nierenerkrankung}

\section{Ernährung}

Die Entwicklung und Progression einer diabetischen Nierenerkrankung kann durch Optimierung der Blutzucker- und Blutdruckeinstellung sowie durch Vermeidung von Zigarettenrauchen zumindest verlangsamt werden. Hinsichtlich der Eiweißzufuhr mit der Nahrung werden nach den KDIGO-Leitlinien 0,8 g/ kg Körpergewicht sowie die Vermeidung der Überschreitung von 1,3g/kg Körpergewicht empfohlen [33]. Zusätzlich wird noch eine kochsalzarme Diät vorgeschlagen [33]. Es sollte dazu angemerkt werden, dass - obwohl bisher nicht in den Leitlinien angemerkt - je eine Studie bei DM1- und bei DM2-Patienten kritisch die Kochsalzrestriktion hinterfragen, da Hinweise für höhere Mortalität und Progression zur terminalen Niereninsuffizienz bestehen [41, 42]. Dies bedarf aber entsprechender weiterer Studien, bevor Empfehlungen hinsichtlich bestimmter Mengen gemacht werden können.

Neben der allgemeinen Empfehlung wie gesunder Lebensstil werden auch verschiedene Diäten diskutiert, die einen Benefit insbesondere bei kardiovaskulären Risikopatienten erzielen sollen (mediterrane Diät [43]; Dietary Approaches to Stop Hypertension (DASH)-Diät [44]). Ob eine entsprechende Diät die Progression einer Albuminurie oder der diabetischen Nierenerkrankung, kardiovaskuläre Ereignisse oder Gewichtsmanagement beeinflusst, ist unklar [29].

Gewichtsreduktion bei morbider Adipositas durch ein bariatrisch chirurgisches Vorgehen wird immer wieder diskutiert, zumal es Hinweise darauf gibt, dass sich verschiedene metabolische Parameter bei DM2 günstig entwickeln, und das Risiko für mikrovaskuläre Komplikationen reduziert werden kann [45]. Die Option eines bariatrisch-chirurgischen Eingriffes muss im Einzelfall entschieden werden.

\section{Kardiovaskuläres Risiko}

Bei bestehendem DM ist bei Nierenerkrankung konsistent eine substanzielle Erhöhung der Mortalität beobachtet worden [14]. Ein Großteil der erhöhten Mortalität ist auf kardiovaskuläre Erkrankungen zurückzuführen, obwohl die nichtkardiovaskuläre Mortalität ebenso erhöht ist. Albuminurie und eGFR sind unabhängige und zusätzlich assoziierte Risikofaktoren für kardiovaskuläre Ereignisse, kardiovaskuläre Mortalität und Gesamtmortalität [14]. Sowohl Diabetes als auch chronische Niereninsuffizienz (CKD) haben vergleichbare Inzidenzraten von kardiovaskulären Ereignissen wie Patienten mit manifester koronarer Herzerkrankung [46]. Dies führt zur Empfehlung, dass Pati- enten mit DM, CKD oder diabetischer Nierenerkrankung präventiv hinsichtlich kardiovaskulärer Erkrankungen so behandelt werden sollen, als ob sie bereits ein solches Ereignis erlitten hätten [46]. Sowohl bei DM1 als auch bei DM2 zeigten Kohortenstudien, dass das erhöhte Risiko für kardiovaskuläre Erkrankungen und Mortalität auf Patienten mit evidenter diabetischer Nierenerkrankung limitiert ist, während Patienten ohne Albuminurie und mit normaler eGFR ein vergleichbares Risiko zur nichtdiabetischen Population haben [47-49]. Diese Beobachtungen ziehen nach sich, dass Behandlungsstrategien darauf ausgerichtet sein sollen, das hohe kardiovaskuläre Risiko von Patienten mit diabetischer Nierenerkrankung abzuschwächen, um das Überleben zu verbessern [29]. Der Mechanismus durch welchen die diabetische Nierenerkrankung eine kardiovaskuläre Erkrankung beeinflusst, umfasst genauso traditionelle Risikofaktoren (Hyperglykämie, Hypervolämie und Hypertonie, Lipoproteinmetabolismus, systemische Inflammation, oxidativer Stress und endotheliale Dysfunktion) sowie die Initiierung von Mechanismen spezifisch im Zusammenhang mit der Nierenfunktionseinschränkung (Urämietoxine, Anämie und Störungen des Knochen- und Mineralstoffwechsels) [29]. Diese Überlegungen fließen in die unten angeführten Therapieempfehlungen ein.

\section{Lipidstoffwechsel}

Die diabetische Nierenerkrankung wird durch Störungen des Lipidmetabolismus in Zusammenhang mit einer Abnahme der Nierenfunktion abhängig vom Stadium der CKD begleitet. LDL-Cholesterin ist ein etablierter Risikofaktor für kardiovaskuläre Erkrankungen in der Allgemeinbevölkerung. Sein prognostischer Wert ist allerdings bei Personen mit eingeschränkter Nierenfunktion aufgrund einer diabetischen Nierenerkrankung eingeschränkt [50]. Das Ausmaß der LDL-Senkung bei der CKD-Population mit Statinen ist vergleichbar mit Personen mit erhaltener Nierenfunktion [51]. Klinische Untersuchungen bei nicht-dialysepflichtiger CKD gehen davon aus, dass kardiovaskuläre Ereignisse und Mortalität durch Statine bzw. die Kombination Statine/Ezetimibe im Vergleich zu Plazebo gesenkt werden [51]. Der günstige Effekt scheint nicht durch die An- oder Abwesenheit von Diabetes modifiziert zu sein. Während also der kardiovaskuläre Benefit durch Statine bei CKD gut dokumentiert ist, haben Statine keine progressionsverzögernde Wirkung hinsichtlich der Nierenfunktion [52]. Daher werden auch, basierend auf den rezenten KDIGO-Leitlinien, Statine bei allen diabetischen $\mathrm{Pa}-$ tienten mit nicht-dialysepflichtiger Nierenfunktionseinschränkung empfohlen [53]. Rezente Daten haben auch gezeigt, dass PCSK9-Hemmer bei Personen im CKD-Stadium 3 (mit und ohne DM2) vergleichbare lipidsenkende Wirkungen und Sicherheitsprofile 
wie bei Patienten mit einer eGFR $>60 \mathrm{ml} / \mathrm{min} / 1,73 \mathrm{~m}^{2}$ aufweisen [54].

\section{Betreuung der Patienten mit diabetischer Nierenerkrankung}

Eine nephrologische Begutachtung ist bei Unklarheit über die Ätiologie der Nierenerkrankung oder aber fortgeschrittener Nierenerkrankung mit Auftreten von Albuminurie Stadium A2 zu empfehlen, insbesondere um die Diagnose einer diabetischen Nierenerkrankung zu sichern und mögliche Differenzialdiagnosen auszuschließen. Ab Stadium G3 sollte eine gemeinsame Betreuung durch Diabetologen und Nephrologen erfolgen und zusätzlich Augenmerk auf mögliche renale Folgeerkrankungen gelegt werden (s. auch Abb. 2, ab Stadium G3a).

$\mathrm{Ab}$ Stadium G4 ist die führende Betreuung durch Nephrologen obligat, insbesondere wegen der erforderlichen Vorbereitung zur Nierenersatztherapie. Dabei ist die Eignung für eine alleinige Nierentransplantation oder eine kombinierte Nieren- und Pankreastransplantation (bevorzugt bei DM1, aber auch in ausgewählten Fällen bei DM2 möglich [55]) zu prüfen. Optimal wäre es, eine präemptive Transplantation (Lebend- oder Post-mortem-Spende) durchzuführen, jedoch ist bei vielen Patienten primär Hämodialyse oder Peritonealdialyse als Ersttherapie erforderlich.

Die Auswahl des Nierenersatzverfahrens (Hämodialyse, Peritonealdialyse, Transplantation) erfolgt nach den speziellen Indikationen und Kontraindikationen, den Patientenpräferenzen und den individuellen Lebensumständen.

\section{Diabeteseinstellung}

Bei DM1 und 2 sollte möglichst eine Optimierung der diabetischen Stoffwechselsituation angestrebt werden. In der Primärprävention sind niedrigere $\mathrm{HbA}_{1 c^{-}}$ Werte zu fordern als in fortgeschrittenen Stadien der CKD und in der Sekundärprävention. Hier hat sich

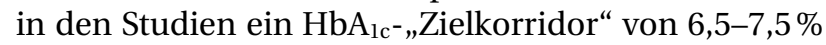
als sinnvoll erwiesen. Unabhängig davon sollte aufgrund der Vorgeschichte, Komorbidität, Hypoglykämieneigung und diabetischer Begleiterkrankungen (Retinopathie, Neuropathie) insbesondere bei älteren Patienten eine individualisierte Festlegung der Therapieziele erfolgen. Bei nachlassender Nierenfunktion ist besonders das erhöhte Risiko der Hypoglykämie zu berücksichtigen. Die Wahl antidiabetischer und anderer Medikamente bedarf bei eingeschränkter Nierenfunktion erhöhter Aufmerksamkeit, da Zulassungseinschränkungen und Kontraindikationen vorliegen können.

\section{Spezielle Aspekte einiger blutzuckersenkender} Medikamente

Einige antihyperglykämische Substanzen haben direkte renale Effekte gezeigt, die sich nicht alleine durch die Blutzuckersenkung erklären lassen [56]. Eine Reihe an kardiovaskulären Outcomestudien untersuchte renale Effekte als sekundäre Outcomeparameter:

Empagliflozin, ein Vertreter der SGLT2-HemmerKlasse, zeigte in der EMPA-REG-OUTCOME-Studie eine signifikante $39 \%$ relative Risikoreduktion im kombinierten Endpunkt „Verschlechterung der Nephropathie“, bestehend aus Progression zu Makroalbuminurie, Verdopplung des Serumkreatinins, Beginn einer Nierenersatztherapie oder Tod renaler Ursache [57]. Auch wenn der Neubeginn einer Empagliflozin-Therapie bei einer eGFR von $<60 \mathrm{ml} / \mathrm{min} / 1,73 \mathrm{~m}^{2}$ nach derzeitiger Fachinformation aufgrund der nachlassenden blutzuckersenkenden Wirkung [58] nicht mehr empfohlen wird, zeigten die EMPA-REG-OUTCOME-Daten, dass der signifikante Benefit der Substanz, der hinsichtlich der kardiovaskulären Ereignisse, des kardiovaskulären Todes, der Gesamtmortalität und der Hospitalisierung wegen Herzinsuffizienz gezeigt wurde, in der Gruppe jener mit einer eGFR $<60 \mathrm{ml} / \mathrm{min} / 1,73 \mathrm{~m}^{2}$ ebenfalls vorliegt [59].

Auch die weiteren kardiovaskulären Outcomestudien mit den SGLT-2-Hemmern Canagliflozin und Dapagliflozin konnten die Reduktion im kombinierten renalen Endpunkt bestätigen [60, 61].

Von den Vertretern der GLP-1-Rezeptoragonisten hat Liraglutide in der LEADER-Studie den kombinierten renalen Endpunkt (persistierende UACR $>300 \mathrm{mg} / \mathrm{g}$, Verdopplung des Serumkreatinins, terminale Niereninsuffizienz oder Tod aufgrund terminaler Niereninsuffizienz) um relative $22 \%$ im Vergleich zur Plazebogruppe (i. e. Blutzuckersenkung ohne GLP-1RA) reduziert, ein Effekt, der in erster Linie durch eine Reduktion im Auftreten von Makroalbuminurie getragen wird [62]. Semaglutide bestätigte diesen Effekt in der SUSTAIN-6-Studie [63].

\section{Therapiebesonderheiten bei nachlassender Nierenfunktion}

Die Auswahl von oralen Antidiabetika hat in den letzten Jahren deutlich zugenommen und gestaltet sich aufgrund der Nierenfunktionseinschränkung schwieriger als bei diabetischen Patienten mit normaler Nierenfunktion [64]. Ebenso ist auf die erhöhte Hypoglykämieneigung in diesem Zusammenhang Aufmerksamkeit zu legen [65]. Im Folgenden werden die wesentlichen Substanzen bzw. Substanzgruppen aufgelistet:

- Metformin galt nach früheren Befunden aufgrund seiner Plasmaeliminationshalbwertszeit von 4,0-8,7 h [66], kompletter renaler Elimination und einer möglichen Gefahr einer Laktatazidose bei ein- 
geschränkter Nierenfunktion als kontraindiziert bei mittel- bis höhergradiger Nierenfunktionseinschränkung. Dies wurde in den letzten Jahren jedoch kontrovers diskutiert, da die Evidenz aus der klinischen Praxis fehlt $[67,68]$. Die FDA und EMA haben 2016 die Empfehlungen für die Verwendung von Metformin daher geändert. Metformin ist demnach bei einer eGFR $<30 \mathrm{ml} / \mathrm{min} / 1,73 \mathrm{~m}^{2}$ kontraindiziert, unter einer eGFR von $45 \mathrm{ml} / \mathrm{min} / 1,73 \mathrm{~m}^{2}$ sollte Metformin nicht neu begonnen werden, die Dosis bei bestehender Therapie auf $1000 \mathrm{mg}$ am Tag beschränkt und der Patient wie seine eGFR engmaschiger überwacht werden. Rezente Studien zur Verwendung von Metformin bei eingeschränkter Nierenfunktion unterstützen diese Vorgangsweise [69] und empfehlen im Stadium 3b die Aufteilung der 1000-mg-Tagesmaximaldosis auf 2-mal täglich $500 \mathrm{mg}$ [70].

Sulfonylharnstoffe (SH) stellen aufgrund des Hypoglykämierisikos nicht das optimale orale Antidiabetikum bei Patienten mit CKD dar. Zwischen den einzelnen Substanzen gibt es erhebliche Unterschiede. Es sollte auf die Gabe des vorwiegend renal eliminierten Glibenclamid verzichtet werden (heutzutage kaum mehr verwendet) wegen der $\mathrm{Ku}$ mulationsgefahr mit Neigung zu schwerer und protrahierter Hypoglykämie. Gliclazid sollte bei CKD in niedriger Dosierung begonnen und alle 4 Wochen dosistitriert werden. Glimepirid kann im Stadium CKD G1-3 in normaler Dosis, im Stadium G4 reduziert $(1 \mathrm{mg} / \mathrm{Tag})$ verabreicht werden und sollte im Stadium G5 vermieden werden [71]. Das Hypoglykämierisiko erscheint am niedrigsten bei Gliclazid [72], gefolgt von Glipizid und Glimepirid [73]. Dennoch ist insgesamt das Hypoglykämierisiko unter SH 10-fach so hoch wie unter Metformin und 4- bis 5-fach höher als unter Pioglitazon [74-77].

- Bei Verwendung von Repaglinid kann bis Stadium G4 ohne Dosisreduktion vorgegangen werden. Für Repaglinid gibt es im Stadium G5 keine Erfahrung.

- Pioglitazon als einzig verbleibender Vertreter der Thiazolidindione muss nicht dosisreduziert werden [71]. Pioglitazon kann entsprechend der Fachinformation bei einer Kreatinin-Clearance $>4 \mathrm{ml} / \mathrm{min}$ eingesetzt werden. Der Verdacht auf eine gering höhere Rate an Blasenkarzinomen wird noch immer kontroversiell diskutiert [78, 79]. Die Substanz Rosiglitazon wurde aufgrund kardiovaskulärer Sicherheitsbedenken in Europa vom Markt genommen $[80,81]$. In einer Metaanalyse wurde auch noch gezeigt, dass die Behandlung mit Glitazonen bei Frauen $\mathrm{zu}$ einer höheren Frakturrate führen kann, sowohl bei Pioglitazon als auch bei Rosiglitazon [82]. Dieser Effekt war aber nach Absetzen der Substanzen wiederum abgeschwächt [83]. Für Pioglitazon werden auch mögliche nephroprotektive Mechanismen (insbesondere antialbuminurische) disku- tiert [74, 76, 77]. Die Datenlage ist aber insgesamt schwach [56].

- Der Glukosidasehemmer Acarbose kann grundsätzlich in allen Stadien der CKD gegeben werden, ab Stadium G4 jedoch nur in seiner niedrigsten Dosis (<50 mg) [71].

- Für GLP-1-Analoga gilt: Exenatid 1-mal wöchentlich sollte nach aktueller Datenlage bei Patienten mit einer eGFR $<50 \mathrm{ml} / \mathrm{min} / 1,73 \mathrm{~m}^{2}$ nicht eingesetzt werden. Liraglutid kann bis $\mathrm{zu}$ einer eGFR $>15 \mathrm{ml} / \mathrm{min} / 1,73 \mathrm{~m}^{2}$ ohne Dosisanpassung angewendet werden [84], für Dulaglutide und Lixisenatide ist bis $\mathrm{zu}$ einer eGFR von $30 \mathrm{ml} / \mathrm{min} / 1,73 \mathrm{~m}^{2}$ ebenfalls keine Dosisanpassung notwendig (aktuelle Fachinformationen).

- Für DPP-4-Hemmer gilt: Linagliptin kann in allen Stadien ohne Dosisanpassung gegeben werden, da es primär hepatobiliär ausgeschieden wird. Bei anderen DPP-4-Hemmern wie Sitagliptin, Vildagliptin, Saxagliptin und Alogliptin sind ab Stadium G3 Dosisanpassungen erforderlich [71]. Es werden auch mögliche nephroprotektive Mechanismen dieser Substanzgruppe diskutiert [77, 85-87]. $\mathrm{Zu}$ beachten bleiben auch die Kontraindikationen bei zunehmender Zahl an Kombinationspräparaten mit Metformin.

- SGLT-2-Hemmer: Empagliflozin, Canagliflozin und Dapagliflozin sollten nach derzeitigem Wissensstand unterhalb einer eGFR von $60 \mathrm{ml} / \mathrm{min}$ nicht begonnen werden und spätestens ab einer eGFR von $45 \mathrm{ml} / \mathrm{min}$ abgesetzt werden [71]. Die positiven kardiovaskulären Effekte wurden bereits oben angeführt [61, 88, 89].

- Bei Insulinen ist auf eine mögliche Dosisreduktion in Abhängigkeit von der Nierenfunktionseinschränkung zu achten.

\section{Blutdruckeinstellung}

Eine antihypertensive Behandlung von Diabetespatienten hat das Ziel, Auftreten und Progression einer diabetischen Nierenerkrankung sowie makrovaskuläre Komplikationen und vorzeitigen kardiovaskulären Tod zu vermeiden. Daraus ergeben sich folgende Therapieziele: Verhinderung des Auftretens bzw. Rückbildung einer Albuminurie; Erhalt der Nierenfunktion; Verhinderung der terminalen Niereninsuffizienz; Reduktion kardiovaskulärer Morbidität und Mortalität. Blutdruckziele für Kinder werden im pädiatrischen Kapitel erläutert.

Basierend auf den rezenten Joint National Committee (JNC) 8 und KDIGO-Leitlinien [90, 91], wird der Zielblutdruck mit $<140 / 90 \mathrm{mmHg}$ bei diabetischer Nierenerkrankung angegeben und auch von der American Diabetes Association und den European Best Practice Guidelines so übernommen [29, 92], um die kardiovaskuläre Mortalität und die Progression der CKD zu reduzieren. Zusätzlich wird von KDIGO 
bei einer Albuminurie $>30 \mathrm{mg} / \mathrm{g}$ ein Zielblutdruck von $<130 / 80 \mathrm{~mm} \mathrm{Hg}$ vorgeschlagen [91]. Eine Unterstützung für diese Zielwerte ergibt sich aus einer limitierten Anzahl von randomisierten Studien, welche auch Patienten mit Diabetes beinhalteten und sich auf kardiovaskuläre Ereignisse fokussierten [29]. Allerdings existieren keine randomisierten Studien hinsichtlich Zielblutdruckwerten, die auf renale Ereignisse eingehen. Daten, welche eine Progressionsverzögerung der CKD zeigen, stammen ausschließlich von 3 randomisierten Studien bei Patienten ohne diabetischer Nierenerkrankung, welche Afroamerikaner mit hypertensiver Nephropathie, Patienten mit IgA-Nephritis und Patienten mit CKD ohne spezifische Diagnose umfassten [93].

Während die Daten derzeit insuffizient sind, niedrigere systolische Blutdruckwerte bei Personen mit diabetischer Nierenerkrankung anzustreben, gab es ein Signal aus klinischen Studien, dass diastolische Blutdruckwerte $<70 \mathrm{~mm} \mathrm{Hg}$ und insbesondere $<60 \mathrm{~mm} \mathrm{Hg}$ bei älteren Patienten problematisch sind [94]. Daten von Patienten mit Stadium G3 oder höher zeigten, dass ein diastolischer Blutdruckwert $<60 \mathrm{mmHg}$ mit einer erhöhten Inzidenzrate an terminaler Niereninsuffizienz vergesellschaftet ist [95], während andere Studien bei Patienten ohne CKD bei diastolischen Werten $<65 \mathrm{~mm} \mathrm{Hg}$ eine Assoziation mit schlechterem Outcome der kardiovaskulären Erkrankungen zeigten [96].

Ein Benefit durch die Verwendung von Blockern des Renin-Angiotensin-Signalweges, sei es durch Verwendung eines ACE-Hemmers oder Angiotensin-Rezeptorblockers, ist durch eine Fülle von klinischen Daten nachgewiesen, insbesondere hinsichtlich der Reduktion von renalen Ereignissen bei Patienten im Stadium G3 oder höher, solchen mit einer Albuminurie, Hypertonie und Diabetes [5, 97, 98]. Daher stellen diese sicherlich die First-line-Therapie dar, auch wenn es Hinweise gibt, dass andere Antihypertensiva gleichwertig hinsichtlich harter kardiovaskulärer Endpunkte und dem Auftreten von ESRD wären [99]. Das spiegelt sich auch in den europäischen Hypertonieleitlinien wider [100]. Rezent wurde der Focus darauf gelegt, durch eine sog. RAAS-Doppelblockade das Ergebnis bei Patienten mit diabetischer Nierenerkrankung noch weiter zu verbessern. Gegensätzlich zur aufgestellten Hypothese mussten 2 klinische Studien vorzeitig aufgrund von höheren Raten an Hyperkaliämie und/oder akutem Nierenversagen und fehlender Effizienz gestoppt werden [101, 102]. Dies wurde auch noch durch die ONTARGET-Studie bei kardiovaskulären Hochrisikopatienten bestätigt [103].

Sehr häufig sind aber zur Erreichung der Zielblutdruckwerte Kombinationstherapien notwendig. Rezent wurde in der ACCOMPLISH-Studie gezeigt, dass die Kombination eines ACE-Hemmers mit einem Dihydropyridin-Kalziumantagonisten hinsichtlich des kardiovaskulären Outcomes, aber auch der Progressionsverzögerung der Nephropathie einer Kombina- tion mit einem Thiaziddiuretikum überlegen ist [104, 105]. Dies ist umso bemerkenswerter, da in der ACCOMPLISH-Studie $60 \%$ der Studienteilnehmer DM2 aufwiesen.

\section{Zusammenfassung}

Zielwerte und Maßnahmen bei diabetischer Nierenerkrankung

- $\mathrm{RR}<140 / 90 \mathrm{~mm} \mathrm{Hg}$

- $\mathrm{RR}<130 / 80 \mathrm{mmHg}$ bei Albuminurie (Stadium A2 und A3)

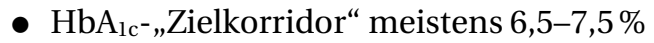
(48-58 mmol/mol) (bei fortgeschrittener CKD)

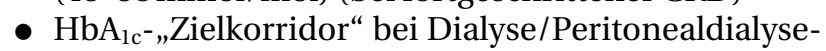
patienten $7-8,0 \%(53-64 \mathrm{mmol} / \mathrm{mol})$

- LDL-Cholesterol-Ziel:

- Zumindest $<100 \mathrm{mg} / \mathrm{dl}$ für die meisten Patienten (für Details s. Lipide: Diagnostik und Therapie bei Diabetes mellitus)

- Bei CKD G3 und G4 <70 mg/dl

- Hämoglobin 9-11 g/dl (eGFR Stadium CKDG 4-5)

- Elektrolyte im Normbereich

- Normalisierung der Eiweißzufuhr auf täglich 0,8 g/kg bis $1,3 \mathrm{~g} / \mathrm{kg}$ Körpergewicht

- Thrombozytenaggregationshemmer (Abwägung des potentiellen kardiovaskulären Benefits gegenüber dem Blutungsrisiko)

- Verzicht auf Rauchen

- Exakte Nutzen-Risiko-Abwägung vor Einsatz potenziell nephrotoxischer Medikamente (z.B. nichtsteroidale Antirheumatika, bestimmte Antibiotika)

- Protektive Maßnahmen bei Röntgenkontrastmittelgabe wegen der erhöhten Gefahr eines akuten Nierenversagens (CT mit KM: bei eGFR $<30 \mathrm{ml}$; bei arteriellen Angiographien eGFR $<45 \mathrm{ml}$ ): auf ausreichende Hydrierung achten (http://www.esur-cm. org/index.php/en/)

- Multifaktorielles Risikofaktorenmanagement

- Beachten der möglichen Kumulation von Begleitmedikamenten

- Beachten des erhöhten kardiovaskulären Risikos mit Screening für Angiopathie

- Beachten von Harnwegsinfekten (Restharn?) und adäquate Antibiotikatherapie

\section{Kontrollen bei Patienten mit diabetischer} Nierenerkrankung

- Je nach CKD-Stadium und Progression mindestens 2- bis 4-mal jährliche Kontrollen

- $\mathrm{HbA}_{\mathrm{lc}}$, Lipide

- Bestimmung der Albuminurie bzw. Albumin-Kreatinin-Ratio

- Bestimmung der Retentionsparameter und Serumelektrolyte (Kreatinin, Harnstoff oder BUN, Kalium) 
- Bestimmung der eGFR, bei Unklarheiten KreatininClearance

- Blutdruckselbstmessung mit Protokollierung, empfohlen ambulante 24-h-Blutdruckmessung

Bei einer Kreatinin-Clearance $<60 \mathrm{ml} / \mathrm{min}$ zusätzlich:

- Blutbild

- Eisenstatus mit Ferritin, Transferrin, Transferrinsättigung, Serumeisen

- Serumphosphat, Serumkalzium

- Parathormon, 25-OH Vitamin D

- Bestimmung der venösen Blutgase insbesondere bei eGFR $<30 \mathrm{ml} / \mathrm{min}$

- Cave: Hyperkaliämie beim Einsatz von RAAS-blockierenden Antihypertensiva wie ACE-Hemmern, ARB sowie auch Aldosteronantagonisten

- Interdisziplinäre diabetologisch-nephrologische Betreuung ab eGFR $<60 \mathrm{ml} / \mathrm{min}$ (Stadium G3)

- Therapieführung durch den Nephrologen - Vorbereitung auf Nierenersatztherapie oder Transplantation ab einer eGFR $<30 \mathrm{ml} / \mathrm{min}$ (Stadium G4)

- Hepatitis-B-Virus-Impfschutz

- Bei Auftreten einer akuten Niereninsuffizienz bzw. Verdacht auf das Vorliegen einer nichtdiabetischen Nierenerkrankung ist eine umgehende nephrologische Begutachtung des Patienten zu veranlassen

Funding Open access funding provided by Medical University of Graz.

Interessenkonflikt $\mathrm{H}$. Sourij hat von folgenden Unternehmen, die auch fördernde Mitglieder der ÖDG sind, Forschungsunterstützungen und/oder Honorare erhalten: Amgen, AstraZeneca, Boehringer-Ingelheim, Eli Lilly, Merck Sharp \& Dohme, Novo Nordisk, Sanofi-Aventis, Takeda. R. Edlinger hat Honorare für Vorträge in den letzten Jahren von folgenden Firmen erhalten: AstraZeneca, Boehringer Ingelheim, Merck Sharp \& Dohme, Takeda, Novartis und SanofiAventis. Betreff der Leitlinien hat R. Edlinger keine unmittelbaren Interessenkonflikte anzugeben. F.C. Prischl hat (während seiner beruflichen Laufbahn) Honorare für Vorträge, Reiseunterstützungen oder Honorare als Mitglied von Advisory Boards von AstraZeneca, Bayer, Boehringer Ingelheim, Eli Lilly, Janssen-Cilag, Novartis, Roche, Takeda erhalten. S. Kaser hat von folgenden Unternehmen, die auch fördernde Mitglieder der ÖDG sind, Forschungsunterstützungen und/oder Honorare erhalten: AstraZeneca, Boehringer Ingelheim, Eli Lilly, Merck Sharp \& Dohme, Novartis, Novo Nordisk, Sanofi-Aventis, Servier, Takeda. S. Horn hat von folgenden Unternehmen Vortragshonorare erhalten: Servier, Novartis, Boehringer-Ingelheim. Betreff der Leitlinien hat S. Horn keine unmittelbaren Interessenkonflikte anzugeben. B. Paulweber hat in den letzten Jahren von folgenden Unternehmen Forschungsunterstützungen und/oder Honorare für Vortrags- und/oder Beratungstätigkeiten erhalten: Abbott, Amgen, AstraZeneca, Boehringer Ingelheim, Bristol Myers Squibb, Bayer, Eli Lilly, Merck Sharp \& Dohme, Mylan, Novartis, Novo Nordisk, Pfizer, Sanofi-Aventis, Takeda. Betreff der Leitlinien besteht kein Interessenkonflikt. A. Kautzky-Willer hat von folgenden Unternehmen, die auch fördernde Mitglieder der ÖDG sind, Forschungsunterstützungen/Honorare erhalten: Boehringer Ingelheim, Eli Lilly, Novo Nordisk, Sanofi-Aventis. M. Säemann hat von folgen- den Unternehmen Forschungsunterstützungen und/oder Honorare erhalten: Amgen, Astellas, Boehringer-Ingelheim, Chiesi, Novartis, Otsuka, Vifor. M. Clodi hat von folgenden Unternehmen, die auch fördernde Mitglieder der ÖDG sind, Forschungsunterstützungen und/oder Honorare erhalten: AstraZeneca, Boehringer Ingelheim, Eli Lilly, Merck Sharp \& Dohme, Novartis, Novo Nordisk, Sanofi-Aventis, Servier, Takeda. M. Auinger, R. Prager, G. Schernthaner, G. Mayer, R. Oberbauer und A.R. Rosenkranz geben an, dass kein Interessenkonflikt besteht.

Open Access Dieser Artikel wird unter der Creative Commons Namensnennung 4.0 International Lizenz (http:// creativecommons.org/licenses/by/4.0/deed.de) veröffentlicht, welche die Nutzung, Vervielfältigung, Bearbeitung, Verbreitung und Wiedergabe in jeglichem Medium und Format erlaubt, sofern Sie den/die ursprünglichen Autor(en) und die Quelle ordnungsgemäß nennen, einen Link zur Creative Commons Lizenz beifügen und angeben, ob Änderungen vorgenommen wurden.

\section{Literatur}

1. KramarR.AustrianDialysis and TransplantRegistry. Annual report 2017. Accessible via https:/ / www.nephro.at (accessed $8^{\text {th }}$ December 2018)

2. Prischl FC, Auinger M, Saemann M, et al. Diabetes-related end-stage renal disease in Austria 1965-2013. Nephrol Dial Transplant. 2015;30(11):1920-7.

3. Nathan DM, Group DER. The diabetes control and complications trial/epidemiology of diabetes interventions and complications study at 30 years: overview. Diabetes Care. 2014;37(1):9-16.

4. de Boer IH, Afkarian M, Rue TC, et al. Renal outcomes in patients with type 1 diabetes and macroalbuminuria. J Am Soc Nephrol. 2014;25(10):2342-50.

5. Brenner BM, Cooper ME, de Zeeuw D, et al. Effects of losartan on renal and cardiovascular outcomes in patients with type 2 diabetes and nephropathy. $\mathrm{N}$ Engl J Med. 2001;345(12):861-9.

6. Heart Outcomes Prevention Evaluation Study Investigators. Effects of ramipril on cardiovascular and microvascular outcomes in people with diabetes mellitus: results of the HOPE study and MICRO-HOPE substudy. Lancet. 2000;355(9200):253-9.

7. Kainz A, Hronsky M, Stel VS, et al. Prediction of prevalence of chronic kidney disease in diabetic patients in countries of the European Union up to 2025. Nephrol Dial Transplant. 2015;30(Suppl4):iv113-iv8.

8. Zelnick LR, Weiss NS, Kestenbaum BR, et al. Diabetes and CKD in the United States Population, 2009-2014. Clin J Am Soc Nephrol.2017;12(12):1984-90.

9. Haller H, Ito S, Izzo JL Jr., et al. Olmesartan for the delay or prevention of microalbuminuria in type 2 diabetes. NEngl J Med.2011;364(10):907-17.

10. Ruggenenti P, Fassi A, Ilieva AP, et al. Preventing microalbuminuria in type 2 diabetes. $\mathrm{N}$ Engl $\mathrm{J}$ Med. 2004;351(19):1941-51.

11. Atkins RC, BrigantiEM, LewisJB, etal. Proteinuriareduction and progression to renal failure in patients with type 2 diabetes mellitus and overt nephropathy. Am J Kidney Dis. 2005;45(2):281-7.

12. Makino H, Haneda M, Babazono T, et al. Prevention of transition from incipient to overt nephropathy with telmisartan in patients with type 2 diabetes. Diabetes Care. 2007;30(6):1577-8. 
13. Adler AI, Stevens RJ, Manley SE, et al. Development and progression of nephropathy in type 2 diabetes: the United Kingdom Prospective Diabetes Study (UKPDS 64). Kidney Int. 2003;63(1):225-32.

14. Fox CS, Matsushita K, Woodward M, et al. Associations of kidney disease measures with mortality and end-stage renal disease in individuals with and without diabetes: a metaanalysis. Lancet. 2012;380(9854):1662-73.

15. Kimmelstiel P, Kim OJ, Beres J. Studies on renal biopsy specimens, with the aid of the electron microscope. I. Glomeruli in diabetes. Am J Clin Pathol. 1962;38:270-9.

16. Kimmelstiel P, Wilson C. Intercapillary Lesions in the Glomeruli of the Kidney. Am JPathol. 1936;12(1):83-98.7.

17. Mogensen CE, Christensen CK, Vittinghus E. The stages in diabetic renal disease. With emphasis on the stage of incipient diabetic nephropathy. Diabetes. 1983;32(Suppl 2):64-78.

18. Mauer SM, Steffes MW, Ellis EN, Sutherland DE, Brown DM, Goetz FC. Structural-functional relationships in diabetic nephropathy. JClin Invest. 1984;74(4):1143-55.

19. Steinke JM, Mauer M, International Diabetic Nephropathy Study G. Lessons learned from studies of the natural history of diabetic nephropathy in young type 1 diabetic patients. Pediatr Endocrinol Rev. 2008;5(Suppl 4):958-63.

20. Auinger M, Edlinger R, Prischl F, et al. Diabetic nephropathy-update 2012. Wien Klin Wochenschr. 2012;124(Suppl 2):42-9.

21. Kramer HJ, Nguyen QD, Curhan G, Hsu CY. Renal insufficiency in the absence of albuminuria and retinopathy among adults with type 2 diabetes mellitus. JAMA. 2003;289(24):3273-7.

22. Retnakaran R, Cull CA, Thorne KI, Adler AI, Holman RR, Group US. Risk factors for renal dysfunction in type 2 diabetes: U.K. Prospective Diabetes Study 74. Diabetes. 2006;55(6):1832-9.

23. Rodriguez-Poncelas A, Garre-Olmo J, Franch-Nadal J, et al. Prevalence of chronic kidney disease in patients with type 2 diabetes in Spain: PERCEDIME2 study. Bmc Nephrol. 2013;14:46.

24. Thomas MC, Macisaac RJ, Jerums G, et al. Nonalbuminuric renal impairment in type 2 diabetic patients and in the general population (national evaluation of the frequency of renal impairment cO-existing with NIDDM [NEFRON] 11). Diabetes Care. 2009;32(8):1497-502.

25. Ismail-Beigi F, Craven T, Banerji MA, et al. Effect of intensive treatment of hyperglycaemia on microvascular outcomes in type 2 diabetes: an analysis of the ACCORD randomised trial.Lancet. 2010;376(9739):419-30.

26. Krolewski AS. Progressive renal decline: the new paradigm of diabetic nephropathy in type 1 diabetes. Diabetes Care. 2015;38(6):954-62.

27. Macisaac RJ, Ekinci EI, Jerums G. Markers of and riskfactors for the development and progression of diabetic kidney disease. Am J Kidney Dis. 2014;63(2 Suppl2):S39-S62.

28. Molitch ME, Adler AI, Flyvbjerg A, et al. Diabetic kidney disease: a clinical update from kidney disease: improving global outcomes. Kidney Int. 2015;87(1):20-30.

29. Tuttle KR, Bakris GL, Bilous RW, et al. Diabetic kidney disease: a report from an ADA Consensus Conference. Am J Kidney Dis. 2014;64(4):510-33.

30. Fioretto P, Mauer M, Brocco E, et al. Patterns of renal injury in NIDDM patients with microalbuminuria. Diabetologia. 1996;39(12):1569-76.

31. Tervaert TW, Mooyaart AL, Amann K, et al. Pathologic classification of diabetic nephropathy. J Am Soc Nephrol. 2010;21(4):556-63.
32. Stanton RC. Clinical challenges in diagnosis and management of diabetic kidney disease. Am J Kidney Dis. 2014;63(2 Suppl2):S3-S21.

33. Group KDIGOKCW. 2012 clinical practice guideline for the evaluation and management of chronic kidney disease. Kidney Int. 2013;3(1):S1-S150.

34. Levey AS, Bosch JP, Lewis JB, Greene T, Rogers N, Roth D. A more accurate method to estimate glomerular filtration rate from serum creatinine: a new prediction equation. Modification of Diet in Renal Disease Study Group. Ann Intern Med. 1999;130(6):461-70.

35. Levey AS, Coresh J, Greene T, et al. Expressing the Modification of Diet in Renal Disease Study equation for estimating glomerular filtration rate with standardized serum creatininevalues. Clin Chem. 2007;53(4):766-72.

36. Levey AS, Stevens LA, Schmid CH, et al. A new equation to estimate glomerular filtration rate. Ann Intern Med. 2009;150(9):604-12.

37. Kalantar-Zadeh K, Amin AN. Toward more accurate detection and risk stratification of chronic kidney disease. JAMA. 2012;307(18):1976-7.

38. White SL, Polkinghorne KR, Atkins RC, Chadban SJ. Comparison of the prevalence and mortality risk of CKD in Australia using the CKDEpidemiology Collaboration (CKDEPI) and Modification of Diet in Renal Disease (MDRD) Study GFR estimating equations: the AusDiab (Australian Diabetes, Obesity and Lifestyle) Study. Am J Kidney Dis. 2010;55(4):660-70.

39. CarvilleS, Wonderling D, Stevens P, Guideline Development G. Early identification and management of chronic kidney diseasein adults: summary of updated NICEguidance.BMJ. 2014;349:g4507.

40. American Diabetes A. 10. Microvascular complications and foot care: standards of medical care in diabetes-2018. Diabetes Care. 2018;41(Suppl 1):S105-S18.

41. Ekinci EI, ClarkeS, Thomas MC, et al. Dietary salt intake and mortality in patients with type 2 diabetes. Diabetes Care. 2011;34(3):703-9.

42. Thomas MC, Moran J, Forsblom C, et al. The association between dietary sodium intake, ESRD, and all-cause mortality in patients with type 1 diabetes. Diabetes Care. 2011;34(4):861-6.

43. Estruch R, Ros E, Salas-Salvado J, et al. Primary prevention of cardiovascular disease with a Mediterranean diet. N Engl JMed. 2013;368(14):1279-90.

44. Sacks FM, Obarzanek E, Windhauser MM, et al. Rationale and design of the Dietary Approaches to Stop Hypertension trial (DASH). A multicenter controlled-feeding study of dietary patterns to lower blood pressure. Ann Epidemiol. 1995;5(2):108-18.

45. Carlsson LMS, Sjoholm K, Karlsson C, et al. Long-term incidence of microvascular disease after bariatric surgery or usual care in patients with obesity, stratified by baseline glycaemic status: a post-hoc analysis of participants from the Swedish ObeseSubjects study. LancetDiabetes Endocrinol. 2017;5(4):271-9.

46. Tonelli M, Muntner P, Lloyd A, et al. Risk of coronary events in people with chronic kidney disease compared with those with diabetes: a population-level cohort study. Lancet. 2012;380(9844):807-14.

47. Afkarian M, Sachs MC, Kestenbaum B, et al. Kidney disease and increased mortality risk in type 2 diabetes. J Am Soc Nephrol.2013;24(2):302-8.

48. Borch-Johnsen K, Andersen PK, Deckert T. The effect of proteinuria on relative mortality in type 1 (insulin-dependent) diabetes mellitus. Diabetologia. 1985;28(8):590-6. 
49. Groop PH, Thomas MC, Moran JL, et al. The presence and severity of chronic kidney disease predicts all-cause mortalityin type 1 diabetes. Diabetes. 2009;58(7):1651-8.

50. Tonelli M, Muntner P, Lloyd A, et al. Association between LDL-C and risk of myocardial infarction in CKD. J Am Soc Nephrol.2013;24(6):979-86.

51. Palmer SC, Craig JC, Navaneethan SD, Tonelli M, Pellegrini F, Strippoli GF. Benefits and harms of statin therapy for persons with chronic kidney disease: a systematic review and meta-analysis. Ann Intern Med.2012;157(4):263-75.

52. Haynes R, Lewis D, Emberson J, et al. Effects of lowering LDL cholesterol on progression of kidney disease. J Am Soc Nephrol. 2014;25(8):1825-33.

53. Wanner C, Tonelli M, Kidney Disease. Improving Global Outcomes Lipid Guideline Development Work Group M. KDIGOClinical PracticeGuidelinefor Lipid Managementin CKD:summary of recommendation statements and clinical approach to the patient. Kidney Int. 2014;85(6):1303-9.

54. Toth PP, Dwyer JP, Cannon CP, et al. Efficacy and safety of lipid lowering by alirocumab in chronic kidney disease. Kidney Int. 2018;93:1397. https://doi.org/10.1016/j.kint. 2017.12.011.

55. Margreiter C, Resch T, Oberhuber R, et al. Combined pancreas-kidney transplantation for patients with end-stage nephropathy caused by type-2 diabetes mellitus. Transplantation. 2013;95(8):1030-6.

56. Prischl FC, Wanner C. Renal outcomes of antidiabetic treatment options for type 2 diabetes-a proposed MARE definition. Kidney Int Rep. 2018; https://doi.org/10.1016/j. ekir2018.04.008.

57. Wanner C, Inzucchi SE, Zinman B. Empagliflozin and progression of kidney disease in type 2 diabetes. $N$ Engl J Med. 2016;375(18):1801-2.

58. Dekkers CCJ, Wheeler DC, Sjostrom CD, Stefansson BV, Cain V, Heerspink HJL. Effects of the sodium-glucose cotransporter 2 inhibitor dapagliflozin in patients with type 2 diabetes and Stages 3b-4 chronic kidney disease. Nephrol Dial Transplant.2018; https://doi.org/10.1093/ndt/gfx350.

59. Wanner C, Lachin JM, Inzucchi SE, et al. Empagliflozin and clinical outcomes in patients with type 2 diabetes mellitus, established cardiovascular disease, and chronic kidney disease. Circulation. 2018;137(2):119-29.

60. Neal B, Perkovic V, Mahaffey KW, et al. Canagliflozin and cardiovascular and renal events in type 2 diabetes. $\mathrm{N}$ Engl J Med. 2017;377(7):644-57.

61. Wiviott SD, Raz I, Bonaca MP, et al. Dapagliflozin and Cardiovascular Outcomes in Type 2 Diabetes. NEJM. 2018. https:// doi.org/10.1056/NEJMoa1812389

62. Marso SP, Daniels GH, Brown-Frandsen K, et al. Liraglutide and cardiovascular outcomes in type 2 diabetes. $\mathrm{N}$ Engl J Med. 2016;375(4):311-22.

63. Marso SP, Bain SC, Consoli A, et al. Semaglutide and cardiovascular outcomes in patients with type 2 diabetes. N Engl J Med.2016;375(19):1834-44.

64. Schernthaner G, Schernthaner GH. Current treatment of type 2 diabetes. Internist (Berl). 2012;53(12):1399-410.

65. Alsahli M, Gerich JE. Hypoglycemia in patients with diabetes and renal disease. JClin Med. 2015;4(5):948-64.

66. Scheen AJ. Clinical pharmacokinetics of metformin. Clin Pharmacokinet. 1996;30(5):359-71.

67. Nye HJ, Herrington WG. Metformin: the safest hypoglycaemic agent in chronic kidney disease? Nephron Clin Pract. 2011;118(4):380-3.

68. Pilmore HL. Review: metformin: potential benefits and use in chronic kidney disease. Nephrology (Carlton). 2010;15(4):412-8.
69. Inzucchi SE, Lipska KJ, Mayo H, Bailey CJ, McGuire DK. Metformin in patients with type 2 diabetes and kidney disease: a systematic review. JAMA. 2014;312(24):2668-75.

70. Lalau JD, Kajbaf F, Bennis Y, Hurtel-Lemaire AS, Belpaire F, De Broe ME. Metformin treatment in patients with type 2 diabetes and chronic kidney disease stages 3A, 3B, or 4 . Diabetes Care. 2018;41(3):547-53.

71. Guideline development group. Clinical Practice Guideline on management of patients with diabetes and chronic kidney disease stage $3 \mathrm{~b}$ or higher (eGFR $\langle 45 \mathrm{~mL} / \mathrm{min}$ ). NephrolDial Transplant. 2015;30(Suppl2):iil-142.

72. Chan SP, Colagiuri S. Systematic review and meta-analysis of the efficacy and hypoglycemic safety of gliclazide versus other insulinotropic agents. Diabetes Res Clin Pract. 2015;110(1):75-81.

73. Tayek J. SUR receptor activity vs. incidence of hypoglycaemia and cardiovascular mortality with sulphonylurea therapyfordiabetics. Diabetes ObesMetab.2008;10(11):1128-9. author reply 9-30.

74. Charbonnel BH, Matthews DR, Schernthaner G, Hanefeld M, Brunetti P, Group QS. A long-term comparison of pioglitazone and gliclazide in patients with Type 2 diabetes mellitus: a randomized, double-blind, parallel-group comparison trial. Diabet Med. 2005;22(4):399-405.

75. Hanefeld M, Brunetti P, Schernthaner GH, Matthews DR, Charbonnel BH, Group QS. One-year glycemic control with a sulfonylurea plus pioglitazone versus a sulfonylurea plus metformin in patients with type 2 diabetes. Diabetes Care. 2004;27(1):141-7.

76. Matthews DR, Charbonnel BH, Hanefeld M, Brunetti P, Schernthaner G. Long-term therapy with addition of pioglitazone to metformin compared with the addition of gliclazide to metformin in patients with type 2 diabetes: a randomized, comparative study. Diabetes Metab Res Rev. 2005;21(2):167-74.

77. Schernthaner G, Matthews DR, Charbonnel B, Hanefeld M, Brunetti P, Quartet Study G. Efficacy and safety of pioglitazone versus metformin in patients with type 2 diabetes mellitus: a double-blind, randomized trial. J Clin Endocrinol Metab. 2004;89(12):6068-76.

78. Lewis JD, Habel LA, Quesenberry CP, et al. Pioglitazone use and risk of bladder cancer and other common cancers in persons with diabetes. JAMA. 2015;314(3):265-77.

79. Garry EM, Buse JB, Lund JL, Pate V, Sturmer T. Comparative safety of pioglitazone versus clinically meaningful treatment alternatives concerning the risk of bladder cancer in older US adults with type 2 diabetes. Diabetes Obes Metab. 2018;20(1):129-40.

80. Mitka M. Panel recommends easing restrictions on rosiglitazone despite concerns about cardiovascular safety. JAMA. 2013;310(3):246-7.

81. Woodcock J, Sharfstein JM, Hamburg M. Regulatory action on rosiglitazone by the U.S. Food and Drug Administration. NEngl J Med.2010;363(16):1489-91.

82. Zhu ZN, Jiang YF, Ding T. Risk of fracture with thiazolidinediones: an updated meta-analysis of randomized clinical trials. Bone. 2014;68:115-23.

83. Schwartz AV, Chen H, Ambrosius WT, et al. Effects of TZD use and discontinuation on fracture rates in ACCORD Bone study. J Clin Endocrinol Metab. 2015;100(11):4059-66.

84. Mann JMO, Raz I, Frimer-Larsen H, von Scholten B, Idorn T, Poulter N, LEADER Trial Investigators. Safety of liraglutide vs. placebo in patients with type 2 diabetes and CKD in the LEADER trial. New Orleans: American Society of Nephrology Kidney Week; 2017. 
85. Avogaro A, Fadini GP. The effects of dipeptidyl peptidase-4 inhibition on microvascular diabetes complications. Diabetes Care. 2014;37(10):2884-94.

86. Panchapakesan U, Pollock CA. DPP-4 inhibitors-renoprotection in diabetic nephropathy? Diabetes. 2014;63(6): 1829-30.

87. Schernthaner G, Mogensen CE, Schernthaner GH. The effects of GLP-1 analogues, DPP-4 inhibitors and SGLT2 inhibitors on the renal system. Diab Vasc Dis Res. 2014;11(5):306-23.

88. Fitchett D, Zinman B, Wanner C, et al. Heart failure outcomes with empagliflozin in patients with type 2 diabetes at high cardiovascular risk: results of the EMPA-REG OUTCOME(R) trial. Eur Heart J. 2016;37(19):1526. https://doi. org/10.1093/eurheartj/ehv728.

89. Zinman B, Wanner C, Lachin JM, et al. Empagliflozin, cardiovascular outcomes, and mortality in type 2 diabetes. NEngl J Med. 2015;373(22):2117-28.

90. James PA, Oparil S, Carter BL, et al. 2014 evidence-based guideline for the management of high blood pressure in adults: report from the panel members appointed to the Eighth Joint National Committee (JNC 8). JAMA. 2014;311(5):507-20.

91. Wheeler DC, Becker GJ. Summary of KDIGO guideline. What do we really know about management of blood pressure in patients with chronic kidney disease? Kidney Int. 2013;83(3):377-83.

92. Verbeke F, Lindley E, Van Bortel L, et al. A European Renal Best Practice (ERBP) position statement on the Kidney Disease:Improving GlobalOutcomes (KDIGO) clinical practice guidelinefor themanagement ofblood pressureinnon-dialysis-dependent chronic kidney disease: an endorsement with some caveats for real-life application. Nephrol Dial Transplant. 2014;29(3):490-6.

93. Taler SJ, Agarwal R, Bakris GL, et al. KDOQI US commentary on the 2012 KDIGO clinical practice guideline for management of blood pressure in CKD. Am J Kidney Dis. 2013;62(2):201-13.

94. Messerli FH, Mancia G, Conti CR, et al. Dogma disputed: can aggressively lowering blood pressure in hypertensive patients with coronary artery disease be dangerous? Ann Intern Med. 2006;144(12):884-93.
95. Peralta CA, Norris KC, LiS, etal. Blood pressure components and end-stage renal disease in persons with chronic kidney disease: the Kidney Early Evaluation Program (KEEP). Arch Intern Med.2012;172(1):41-7.

96. Kovesdy CP, Bleyer AJ, Molnar MZ, et al. Blood pressure and mortality in U.S. veterans with chronic kidney disease: a cohort study. Ann Intern Med.2013;159(4):233-42.

97. Lewis EJ, Hunsicker LG, Bain RP, Rohde RD. The effect of angiotensin-converting-enzyme inhibition on diabetic nephropathy. The Collaborative Study Group. N Engl J Med. 1993;329(20):1456-62.

98. Lewis EJ, Hunsicker LG, Clarke WR, et al. Renoprotective effect of the angiotensin-receptor antagonist irbesartan in patients with nephropathy due to type 2 diabetes. N Engl J Med. 2001;345(12):851-60.

99. Bangalore S, Fakheri R, Toklu B, Messerli FH. Diabetes mellitus as a compelling indication for use of renin angiotensin system blockers: systematic review and meta-analysis of randomized trials. BMJ. 2016;352:i438.

100. Mancia G, Fagard R, Narkiewicz K, et al. 2013 ESH/ESC Guidelines for the management of arterial hypertension: the Task Force for the management of arterial hypertension of the European Society of Hypertension (ESH) and of the European Society of Cardiology (ESC). J Hypertens. 2013;31(7):1281-357.

101. Fried LF, Emanuele N, Zhang JH, et al. Combined angiotensin inhibition for the treatment of diabetic nephropathy. NEngl J Med.2013;369(20):1892-903.

102. Parving HH, Brenner BM, McMurray JJ, et al. Cardiorenal end points in a trial of aliskiren for type 2 diabetes. $\mathrm{N}$ Engl J Med.2012;367(23):2204-13.

103. Mann JF, Schmieder RE, McQueen M, et al. Renal outcomes with telmisartan, ramipril, or both, in people at high vascular risk (the ONTARGET study): a multicentre, randomised, double-blind, controlled trial. Lancet. 2008;372(9638):547-53.

104. Bakris GL, Sarafidis PA, Weir MR, et al. Renal outcomes with different fixed-dose combination therapies in patients with hypertension at high risk for cardiovascular events (ACCOMPLISH): a prespecified secondary analysis of a randomised controlled trial. Lancet. 2010;375(9721):1173-81.

105. Jamerson K, Weber MA, Bakris GL, et al. Benazepril plus amlodipine or hydrochlorothiazide for hypertension in high-risk patients. NEngl J Med.2008;359(23):2417-28. 